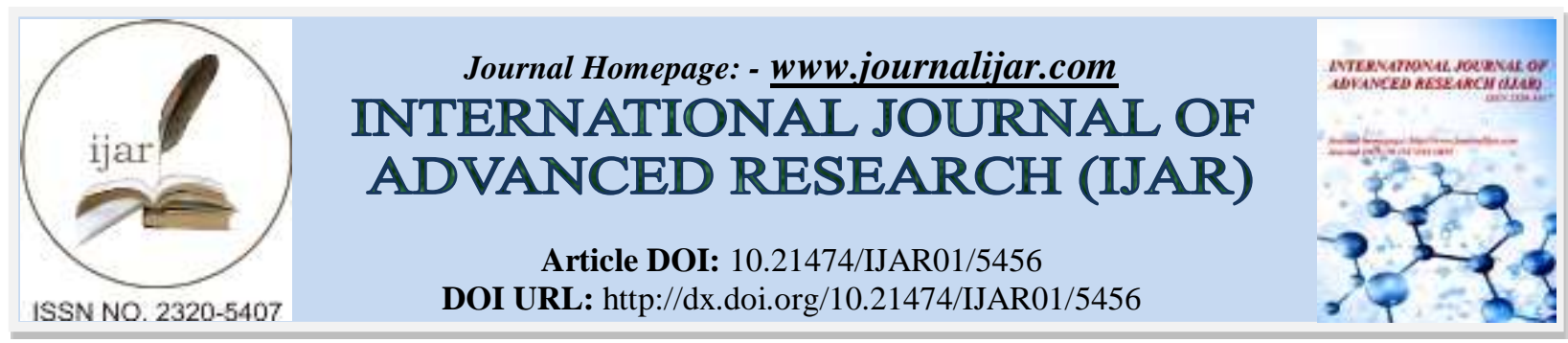

RESEARCH ARTICLE

\title{
PRATIQUES SOCIALES ET SENSIBILISATIONS AUTOUR DE LA QUESTION DES DECHETS URBAINS DANS LE DISTRICT D'ABIDJAN
}

\section{YAO-KOUASSI Quonan Christian ${ }^{1}$ and GOHOUROU Florent ${ }^{2}$.}

1. Enseignant- Chercheur - UFR: Sciences Sociales et Humaines, Université Jean Lorougnon Guédé, (Daloa, Côte d'Ivoire) - Bp 150 Daloa.

2. Maître - Assistant - UFR: Sciences Sociales et Humaines, Université Jean Lorougnon Guédé, (Daloa, Côte d'Ivoire) - Bp 150 Daloa, Chercheur associé à MIGRINTER (CNRS - UMR 7301 - France).

\section{Manuscript Info}

Manuscript History

Received: 17 July 2017

Final Accepted: 19 August 2017

Published: September 2017

Key words:-

Users, Representation, Behavior, Waste,

Communication, Cocody, Yopougon,

Côte d'Ivoire.

\begin{abstract}
The ivory Coast is experiencing strong population growth that has a direct impact on its cleanliness. In order to understand why waste strewn every day on the streets of Abidjan, we were interested in the behavior of the users of the communes of Cocody and Yopougon. The latter are the main actors in the process of sanitation and the management of urban space. On the other hand, our attention has focused on the logics of decision-makers to encourage and encourage users to adopt pro-environmental behaviors. The results of our study show that users find waste to be harmful. However, they do not take good action and contribute to the creation of wild deposits. Their expectations are more focused on the technical aspect of waste management. Finally, the study demonstrates the ineffectiveness of the communication and awareness-raising methods put in place by local and regional authorities to modulate the behavior change of users. The resolution of these various problems requires a regulatory wealth coupled with a pedagogical and permanent communication.
\end{abstract}

Copy Right, IJAR, 2017,. All rights reserved.

\section{Introduction:}

En Côte d'Ivoire, la gestion des déchets ménagers est devenue l'une des principales préoccupations des collectivités territoriales. Cette implication des Autorités locales a une double raison: l'augmentation de l'état d'insalubrité des villes et la pauvreté législative en matière de gestion des déchets des collectivités territoriales qui ne favorise pas la réduction des déchets à la source, leur réutilisation, leur recyclage ainsi que la quantité des déchets résiduels partant en stockage. La prise de conscience globale de l'environnement et de la gestion des déchets par les populations n'émergent pas suffisamment. Toutefois, la bonne gestion des déchets est liée fondamentalement au comportement des populations et de la manière dont ces derniers se débarrassent de leurs déchets. Malheureusement, on constate qu'il existe peu de programmes de sensibilisation cohérents en faveur des usagers afin de modifier leurs comportements quotidiens. En réalité, lorsqu'ils existent, ils sont peu soutenus et apparaissent suite à des crises sanitaires. Il résulte de cette situation l'indifférence et le désintérêt de ces populations vis-à-vis des actions de sensibilisation menées. Dans ce contexte, une meilleure compréhension des stratégies développées par les collectivités territoriales pour impliquer les usagers du service d'élimination des déchets ménagers est indispensable. Cette étude analysera à travers les communes de Yopougon et Cocody, les stratégies de communication mise en œuvre si elles existent. Quelle est la place de la communication et de la sensibilisation dans la transformation des 
mentalités? Quelle est l'intégration des attentes des usagers dans la mise en place des stratégies de gestion des déchets ménagers?

\section{Matériels et Méthode:}

Afin d'analyser les choix des collectivités territoriales, il nous paraît nécessaire au préalable d'identifier les mesures mises en place pour inciter les usagers à adopter des pratiques de gestion des déchets plus respectueuses de l'environnement. La méthodologie utilisée s'appuie sur une recherche documentaire et une prise de connaissance des instruments techniques, tarifaires, informationnels des collectivités. Les différents documents consultés ont été récupérés au sein des différents services techniques des communes et du District. L'existence des mesures d'implications des usagers a été abordée à travers les communes de Cocody et de Yopougon. Notre choix s'est porté sur ces deux communes à cause du contraste et de la stratification sociale très marquée qui en ressort. En effet, Cocody est la commune la plus huppée du district d'Abidjan tandis que Yopougon en est la plus populaire. Ensuite, nous avons effectué une enquête qualitative sur 70 personnes réparties dans ces communes. Les données recueillies ont été traitées à l'aide du logiciel Excel. La plupart des résultats obtenus a été traduite en graphiques ou tableaux en fonction de la pertinence du phénomène qu'on voulait ressortir.

\section{Résultats et discussions:}

\section{1-L'insalubrité à Abidjan: une responsabilité partagée entre Autorités et usagers}

La gestion des déchets a connu plusieurs acteurs et organisations que nous révèlerons dans cette partie à travers la typologie des instruments de gestion mis en place par les collectivités territoriales. Ainsi, nous pourrons mieux cerner le comportement des usagers qui en découle.

\section{1-1-Abidjan et l'insalubrité}

Ces vingt dernières années ont été celles du regain de l'insalubrité à Abidjan. Cette accumulation d'immondices dans la ville d'Abidjan peut se justifier par le bouleversement des modes de vie et le développement d'activités consommatrices. Ces dernières sont liées à l'économie urbaine informelle qui favorise la hausse considérable des déchets dans l'espace public. En effet, que l'on se retrouve à Cocody ou à Yopougon, on observe une concentration de dépôts sauvages. La structure de l'habitat ne serait donc pas le seul déterminant dans la production de déchets. D'autres facteurs importants tels que les nouveaux modes de consommation et le comportement des urbains favorisent la production de déchets qui ont aussi des répercussions sur la salubrité publique. A Abidjan, cette situation est si préoccupante que l'on se croirait parfois dans une véritable ville poubelle. Dans ce contexte, il est nécessaire de connaître le mode de gestion appliqué dans la ville par les collectivités territoriales.

\section{1-2 - L'héritage d'un système de gestion non inclusif}

La gestion des déchets ménagers en Côte d'Ivoire a connu une succession d'acteurs institutionnels. Le passage d'un système de nettoiement à un autre s'est effectué surtout en situation de grande insalubrité. Après les défaillances techniques et organisationnelles du système de gestion mis en place, survient la contestation sociale qui impose le passage à un nouveau système, piloté par de nouveaux acteurs. Abidjan, la capitale économique en a connu plusieurs que l'on peut distinguer en cinq principales périodes:

- la période de 1953 à 1990 ou mode de gestion déléguée, pendant laquelle la gestion ne relevait qu'une seule société privée : la Société Industrielle de Transports Automobiles Africaines (SITAF), filiale de la SITA ;

- la période de 1990 à septembre 1992, pendant laquelle le service des déchets de la ville d'Abidjan et les services de l'Etat se sont occupés de la gestion des déchets ménagers;

- la période de septembre 1992 à 1995, où la gestion a été cédée à une société privée locale, ASH International;

- de 1995 à 2007, ce service a été confié aux services techniques des différentes communes;

- depuis 2007, l'ANASUR (Agence Nationale de la Salubrité Urbaine) est l'organe central de gestion des déchets ménagers. On note également l'existence d'une brigade de salubrité issue du Ministère de l'environnement et du cadre de vie qui a la responsabilité de concevoir le programme national d'élimination des déchets urbains. Le changement d'acteur observé durant les décennies n'a pas profondément modifié les instruments techniques, tarifaires et informationnels utilisés (voir tableau). En effet, notre état des lieux réalisés montre que deux types de mode de collecte impliquant les usagers se dessinent. 
Tableau: Typologie des différents instruments de gestion des déchets ménagers par les collectivités

\begin{tabular}{|l|l|l|l|}
\hline & Instruments techniques & Instruments tarifaires & $\begin{array}{l}\text { Instruments } \\
\text { informationnels }\end{array}$ \\
\hline Mode de collecte 1 & $\begin{array}{l}\text { Collecte en apport } \\
\text { volontaire ; irrégulière de } \\
\text { Fréquence } \\
\text { collecte }\end{array}$ & $\begin{array}{l}\text { Budget général; } \\
\text { TEOM indexée sur les } \\
\text { factures d'électricité } \\
\text { d'informations }\end{array}$ \\
\hline Mode de collecte 2 & $\begin{array}{l}\text { Précollecte en porte à } \\
\text { porte; } \\
\text { Fréquence irrégulière }\end{array}$ & $\begin{array}{l}\text { Budget général; } \\
\text { Payé par les usagers }\end{array}$ & $\begin{array}{l}\text { Pas peu } \\
\text { d'informations }\end{array}$ \\
\hline
\end{tabular}

Source: YAO-KOUASSI

La collecte 1, implique un faible nombre d'usagers dans la gestion des déchets ménagers. Les différents flux de déchets sont en effet collectés en apport volontaire. Ce mode de collecte est généralement employé par les usagers les plus motivés à 23,3\%. Par ailleurs, la fréquence de collecte est irrégulière. La tarification du service repose principalement sur des taxes (budget général, TEOM) qui responsabilisent peu les usagers à l'égard de leur production de déchets. Enfin, les mesures informationnelles sont peu développées car 99,3\% des usagers les trouvent inexistantes. Quant à la collecte 2, elle facilite l'utilisation du service pour les usagers. La majorité des communes, ont choisi ce mode de collecte dans le but d'accompagner le plus grand nombre d'usagers dans le changement de leurs pratiques quotidiennes en simplifiant l'utilisation du service de collecte des déchets ménagers. La majorité des flux de déchets ménagers est collectée en porte-à-porte. Ce procédé facilite la participation des usagers dans le processus de collecte. La fréquence de collecte demeure irrégulière et l'information est quasi inexistante à destination des usagers selon $92 \%$ des enquêtés.

Cette classification représente bien les différents types de stratégie développés par des collectivités territoriales visà-vis des usagers. On constate que le choix de la stratégie relève toujours d'un mode de gestion archaïque qui ne produit aucun résultat probant. Le niveau d'engagement dans le processus de réduction des déchets n'a subi aucune évolution ni transformation des habitudes et comportements des usagers. Ces observations nous amènent à analyser les représentations et les comportements que se font les citadins vis-à-vis de la salubrité.

\section{1-3- Représentation sociale des déchets et comportement des usagers dans l'espace public:}

La ville d'Abidjan s'est étendue avec l'afflux d'une population cosmopolite en quête d'espace vital. Cette population a un impact sur l'espace à Abidjan où chaque type de tissu urbain correspond à une production des déchets. Selon SHADYC-GRILL (2002), cette situation particulière pose un problème de comportement par rapport à l'usage et au partage de l'espace public. Ces citadins continuent à gérer la relation entre l'espace domestique de la cour et l'extérieur (la rue, le chemin ou le mur mitoyen) en fonction d'un « habitus » rural et d'une culture paysanne. Ainsi, la définition de la notion de déchets par les populations ne se relève pas discriminante en fonction de l'appartenance de l'individu à tel ou tel groupe social. En effet, comme le montre la figure ci-dessous, trois typologies se dégagent (inutiles, nuisibles, ordures) que l'on soit à Cocody ou à Yopougon. Nous avons, 25,3\% des enquêtés de Cocody qui qualifient le déchet d'inutile, contre 20,7\% à Yopougon. La majorité des enquêtés définissent le déchet comme étant nuisible à 44,7\% à Cocody et 55,3\% à Yopougon. Enfin, le qualificatif d'ordure qui souligne le dégoût, la puanteur, l'impureté est désigné par 30\% des enquêtés de Cocody et 24,8\% de Yopougon. 


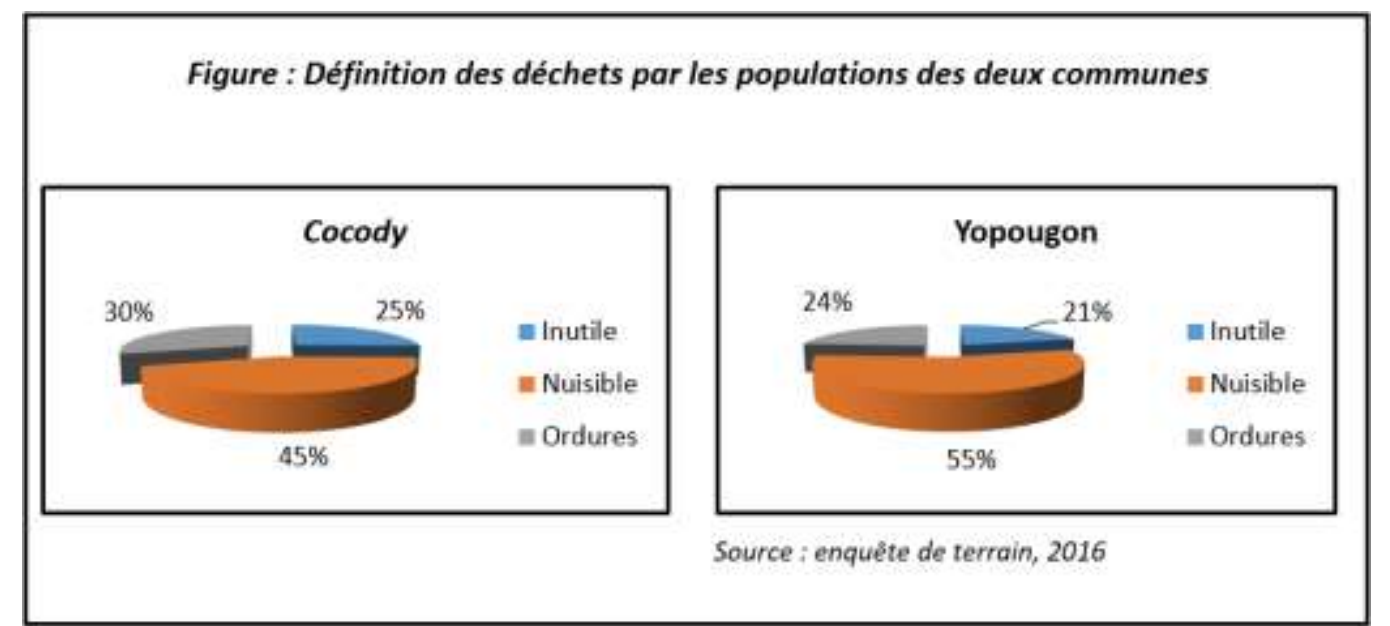

$\mathrm{Au}$ vu des réponses des enquêtés, l'on pourrait croire que le comportement des citoyens serait exemplaire. Tous s'accordent à reconnaitre qu'ils jettent leurs déchets (papiers, sacs plastiques) dans les rues. Comme l'indiquait Zoa (1996), on aurait une certaine image de l'espace public, des routes qui n'appartiennent à personne. Ils sont considérés comme des lieux neutres où les dépôts des ordures ne gêneraient personne. Par ailleurs, les enquêtés incriminent l'état qui pour eux à favoriser ce nouveau comportement et cet incivisme en installant pas/plus de poubelles le long des trottoirs. En effet, ces dernières volées ou vétustes n'ont jamais été remplacées.

\section{2- Attentes des usagers et sensibilisation par la communication}

La gestion des déchets dans une démarche de recherche participative doit intégrer les attentes des usagers, couplées à des campagnes de sensibilisation interpellatrices, rassurantes, attrayantes et stimulantes visant à changer les habitudes vis-à-vis des déchets.

\section{2-1. L'expectative des usagers dans la recherche de solution}

Les attentes des usagers comme nous pouvons le voir à travers le graphe suivant, sont beaucoup plus axées sur la collecte hors de leur zone d'habitat que sur le devenir des déchets une fois collectés. Ainsi, 28\% des personnes interrogées souhaitent que la collecte se fasse dans tous les quartiers quel que soit le niveau social. Ces personnes considèrent que l'état de salubrité observé dans la ville serait imputable aux quartiers défavorisés et que la discrimination en matière de collecte ne devrait plus avoir lieu. $17 \%$ des enquêtés considèrent que le ramassage régulier des ordures (matin et soir), serait une bonne chose au vu des quantités produites par chaque habitant et empêcherait la prolifération des dépôts sauvages. Il revient donc aux collectivités territoriales d'orienter et d'insister auprès des prestataires afin que cette piste soit exploitée. Le premier maillon de la chaine de collecte à savoir les précollecteurs devrait avoir plus de moyens selon 12\% des enquêtés. En effet, ces derniers sont dépourvus d'outils modernes. Les instruments rudimentaires qu'ils utilisent traînent les déchets tout le long de leur parcours en créant des dépôts sauvages. L'essentiel de la stratégie selon les résultats de nos enquêtes doit être basé sur le volet technique. Malheureusement, la prise de conscience des usagers sur leurs attitudes vis-à-vis des déchets qu'ils produisent n'est nullement évoquée par ces derniers. 


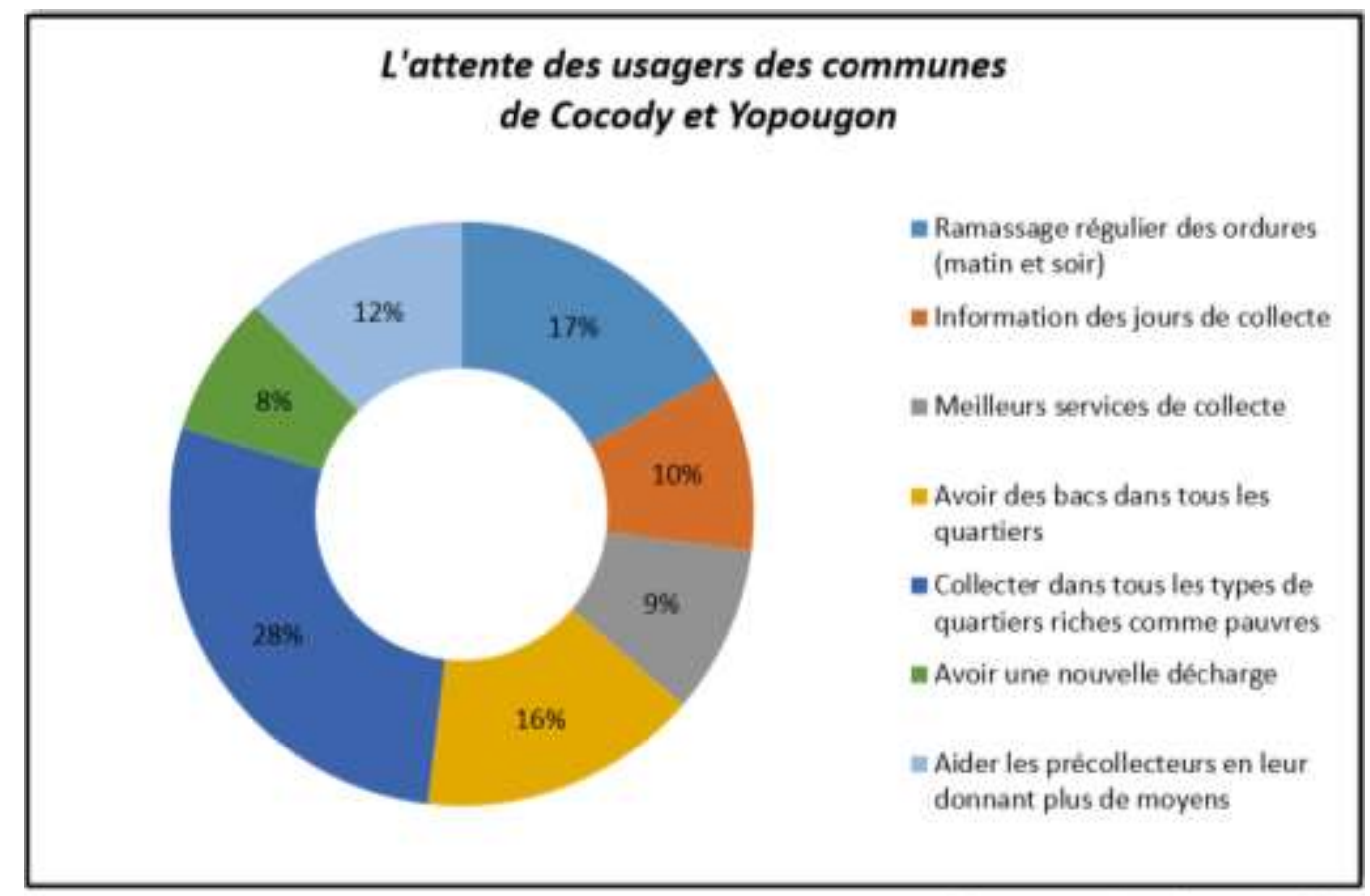

Source : enquête de terrain, 2016

\section{2- Place de la communication et métamorphose des mentalités}

En Côte d'Ivoire, l'audio-visuel est l'unique canal en ce qui concerne l'information sur l'environnement en général. Les autres sources d'information ou de communication sont quasi inexistantes. Nul ne peut réfuter l'impact de la communication sur la transformation des mentalités. Les spécialistes de la publicité et du marketing l'ont si opportunément bien compris qu'ils ne lésinent pas sur les moyens et les stratégies lorsqu'ils doivent imposer leurs nouveaux produits aux consommateurs. Des opérations dénommées «ville propre » dans les années 90 à «grand ménage » aujourd'hui devrait bénéficier d'une approche communicationnelle harmonieuse, élaborée et pédagogique. Cette démarche bien élaborée pendant une bonne période faciliterait l'intégration de concept au travers des messages qui pourront être imprimés dans le subconscient de chaque citoyen.

Notre étude montre que $92 \%$ des enquêtés à Cocody et $99 \%$ de Yopougon révèlent ne pas être informés par la mairie ou le district d'Abidjan. Ces derniers avouent ne pas être également sensibles aux campagnes de communication télévisées. Ils assimilent ces derniers à des faits politiques. En effet, ces campagnes ressemblent plus à de la propagande politique qu'à une mise en avant d'un problème de société qu'on souhaiterait traiter.

Il faut également noter le faible niveau d'instruction d'une partie de la population concernée. Cela pourrait justifier en partie ce manque d'intérêt pour l'information véhiculée. Les mairies se doivent d'assurer l'éducation à environnement, le suivi et la maintenance du cadre de vie de proximité. Ceci lorsque les actions institutionnelles ne sont pas assurées. Pour véritablement réussir les campagnes de sensibilisation, les acteurs de la société civile (ONG, Associations) et les chefs de quartiers doivent être associés comme partenaires de proximité et d'accompagnement. Ces derniers sont plus proches des populations et connaissent mieux leurs logiques et difficultés de fonctionnement.

\section{3-Application des lois en vigueur et réformes:}

La réglementation en matière de déchets n'est pas très fournie ainsi que les textes juridiques spécifiques aux déchets comme on en trouve dans les pays développés. Le législateur s'est penché sur les déchets à travers la loi du 7 juillet 1988. Cette dernière était axée sur les déchets industriels toxiques et nucléaires ainsi que les substances nocives. Pour punir les contrevenants, la loi prévoit une responsabilité civile, pénale et solidaire, ainsi que les sanctions notamment de 1 à 5 ans d'emprisonnement et une amende de 5 millions à 100 millions de Francs CFA ou de l'une de ses peines. Il a fallu attendre huit ans pour voir émerger une loi portant code de l'environnement (Loi n`96-766 du 3 Octobre 1996). Cette loi après avoir posé le principe de la définition des déchets émet d'autres principes: obligation de la collecte ; du traitement et de l'élimination ; obtention d'une autorisation pour l'enfouissement dans le sol ou le sous-sol de déchets non toxiques; respect des normes définies en matière d'élimination des déchets. Nos observations de terrain nous ont permis de remarquer que les différents principes énoncés ne sont pas suffisamment 
ou peu respectés. Les sanctions prévues par les lois ne sont pas appliquées. Ce laxisme motive les entreprises comme les usagers à ne pas avoir un comportement éco-citoyen. Par ailleurs, les collectivités qui ont l'obligation d'élaborer des schémas de collecte et de traitements des déchets selon la réglementation n'en n'ont pas et ne sont pas inquiétés par d'éventuelles sanctions.

Le processus de décentralisation renforcé par l'ordonnance de 2003 a été interrompu par l'ordonnance de 2007. Cette dernière a confié à l'ANASUR (Agence National de la Salubrité Urbaine) toutes les prérogatives en matière de gestion des déchets. Cette centralisation des compétences et prérogatives des collectivités territoriales aggravent la problématique des déchets. En effet, les collectivités territoriales de par leur proximité avec les populations et la production de déchets ménagers sont les mieux placés pour cerner tous les contours de cette problématique dans leur localité. La Brigade de Salubrité Urbaine créée le 4 Février 2008, composante de l'ANASUR a un champ d'action national. Cette dernière comprend deux services: le service de la logistique et des opérations et celui de l'administration générale. Elle est aussi composée des agents des forces de défense et de sécurité affectés aux opérations de terrain et de personnel civil en charge des tâches administratives. La mise en place de cette brigade est de lutter contre les acteurs de pollutions et nuisances ce qui n'est pas toujours le cas.

\section{Conclusion:}

Le déchet est un indicateur objectif des comportements sociaux des usagers dans les collectivités territoriales. Les dépôts sauvages dans les communes d'Abidjan sont ainsi révélateurs des habitudes et du rapport à l'espace qu'ont les usagers. En effet, pour les usagers la notion d'espace public renvoie à un lieu de "non droit" où l'on peut jeter ses déchets sans être inquiété. Certains usagers des communes évoquent comme attente principale le ramassage régulier des dépôts sauvages sans se rendre compte qu'ils sont les premiers à les créer. Les changements d'attitude des usagers ne font pas l'objet de sensibilisation ou de communication adéquate afin que les décideurs et les usagers situent leurs responsabilités et jouent pleinement leur rôle. Il est donc nécessaire de prendre en compte le rapport entre les usagers et leurs collectivités, voire les élus puisque les contestations se manifestent souvent par le rejet des choix opérés par les décideurs. Ces derniers et les usagers doivent faire la preuve de l'intérêt de projet commun car l'un des moteurs puissants d'adhésion des usagers est l'esprit civique, le désir d'agir pour le bien de la communauté. Enfin, la gestion des déchets pose également le problème de pertinence des instances de décisions et des sanctions à appliquer à tout contrevenant à l'insalubrité urbaine.

\section{Références Bibliographiques:}

1. Bertrand J-R et Laurent F., 2003, De la décharge à la déchetterie: questions de géographie des déchets, Presse Universitaire de Rennes, Rennes, 170p.

2. Conseil général de l'économie., 2015, L'économie circulaire ou la compétition pour la ressource, La documentation Française, Paris, 148p.

3. Eyebiyi E., 2010, Gérer les déchets ménagers en Afrique: le Bénin entre local et global, l'Harmattan, Paris, 223p.

4. Gnangui A., 2010, Droit des déchets en Afrique: le cas de la Côte d'Ivoire, l'Harmattan, Paris, 247p.

5. Marcoux M-A, Olivier F et Théry F., 2016, Déchets et économie circulaire: conditions d'intégration pour une valorisation en filières industrielles, Lavoisier Tec \& Doc, Paris, 331p.

6. Najib et al., 2014, Gestion des déchets ménagers dans la ville de Khouribga (Maroc) étude du comportement du citoyen, ScienceLib, Editions Mersenne,23p.

7. N'gnikam E., 2006, Les villes d'Afrique face à leurs déchets. Belfort, Université Technologie de Belfort, Montbéliard, 281p.

8. SHADYC-Marseille et GRIL-Ouagadoudou, 2002, Une anthropologie politique de la frange. Conceptions culturelles, pratiques sociales et enjeux institutionnels de la propreté urbaine à Ouagadougou et à BoboDioulasso. Rapport final Programme de gestion durable des déchets et de l'assainissement urbain, PDM-PS-Eau Paris, septembre, 223p.

9. Tchuikoua L. B., 2015, Gestion des déchets solides ménagers à Douala: acteurs, pratiques urbaines et risques environnemento-sanitaires, l'Harmattan, Paris, $189 \mathrm{p}$.

10. Yao-Kouassi Q. C., 2010, A la recherche d'une synergie pour la gestion des déchets ménagers en Côte d'Ivoire: Cas du district d'Abidjan. Thèse de géographie sociale, Université du Maine au Mans, 305p.

11. Zoa A. S., 1996, Les ordures à Yaoundé: Urbanisation, Environnement et Politique au Cameroun, L'harmattan, paris, 192p. 